PROCEEDINGS OF THE

AMERICAN MATHEMATICAL SOCIETY

Volume 132, Number 8, Pages 2441-2447

S 0002-9939(04)07349-6

Article electronically published on March 25, 2004

\title{
ENLARGING TOTALLY GEODESIC SUBMANIFOLDS OF SYMMETRIC SPACES TO MINIMAL SUBMANIFOLDS OF ONE DIMENSION HIGHER
}

\author{
CLAUDIO GORODSKI
}

(Communicated by Richard A. Wentworth)

\begin{abstract}
We show that a totally geodesic submanifold of a symmetric space satisfying certain conditions admits an extension to a minimal submanifold of dimension one higher, and we apply this result to construct new examples of complete embedded minimal submanifolds in simply connected noncompact globally symmetric spaces.
\end{abstract}

\section{INTRODUCTION}

A submanifold of a Riemannian manifold is called totally geodesic if its second fundamental form vanishes identically, and it is called minimal if the trace of its second fundamental form vanishes identically. Of course, any totally geodesic submanifold is minimal. In this paper, in the presence of enough symmetries in the ambient space, we give a sufficient condition to be able to "extend" a given totally geodesic submanifold to a minimal submanifold of dimension one higher, namely to construct a new minimal submanifold that contains the original totally geodesic submanifold as a hypersurface of codimension one.

In fact, suppose that $S$ is a totally geodesic submanifold of a symmetric space $M$ of codimension greater than one. Let $\gamma$ be a geodesic in $M$ that is normal to $S$ at $\gamma(0) \in S$. The transvections along $\gamma$ define a (local) one-parameter group $\left\{\psi_{t}\right\}$ of (local) isometries of $M$. We describe a condition on the pair $\left(S, \gamma^{\prime}(0)\right)$ which guarantees that the submanifold obtained by translation of $S$ using $\left\{\psi_{t}\right\}$ is minimal (and in general not totally geodesic). Under reasonable global conditions on $M$ and $S$, this result is then extended to produce a minimal embedding of $\mathbf{R} \times S$ into $M$.

The construction can be applied to a variety of totally geodesic submanifolds in symmetric spaces, yielding (mostly new) examples of minimal submanifolds. For example, there is a rather large class of totally geodesic submanifolds in symmetric spaces, the so-called reflective submanifolds (which were introduced and classified by Leung in [Leu73, Leu74, Leu79a]), which can be translated along any normal geodesic to produce a minimal submanifold. It is worth noting here the case of the complex hyperbolic space $\mathbf{H}^{n}(\mathbf{C})$. The maximal reflective submanifolds in $\mathbf{H}^{n}(\mathbf{C})$ turn out to be of two types: complex totally geodesic (congruent to $\mathbf{H}^{n-1}(\mathbf{C})$ ) or

Received by the editors March 10, 2003 and, in revised form, May 13, 2003.

2000 Mathematics Subject Classification. Primary 53C42; Secondary 53C35.

Partially supported by CNPq. 
totally real totally geodesic (congruent to $\mathbf{H}^{n}(\mathbf{R})$ ). It is easy to show that the minimal hypersurface constructed by translating a codimension-two complex totally geodesic submanifold along a normal geodesic is a bisector. Bisectors in $\mathbf{H}^{n}(\mathbf{C})$ are defined as the geometric loci of points equidistant from two distinct given points; they are all congruent and have been used in the construction of fundamental polyhedra for discrete groups of isometries of $\mathbf{H}^{n}(\mathbf{C})$ (see [Gol99] for these concepts). On the other hand, the translation of a totally real totally geodesic submanifold along a normal geodesic produces a new congruence class of minimal submanifolds of $\mathbf{H}^{n}(\mathbf{C})$. In the case $n=2$ these are minimal hypersurfaces, and are expected as well to play an important role in the construction of discrete groups of isometries of $\mathbf{H}^{2}(\mathbf{C})$. The author would like to thank N. Gusevskii for the suggestion to consider this hypersurface, which originated the whole subject of this paper.

\section{The construction}

Theorem 2.1. (a) Let $M$ be a locally symmetric space, $S \subset M$ a totally geodesic submanifold of codimension greater than one, $p \in S$, and $X$ an infinitesimal transvection that extends a nonzero vector $X_{p} \perp T_{p} S$ and whose local oneparameter group of isometries is denoted by $\left\{\psi_{t}\right\}$. Suppose that for every infinitesimal transvection $Y$ at $p$ we have that

$$
\left(L_{X} L_{Y}^{2 n+1} X\right)_{p} \in T_{p} S \quad \text { for } n=0,1,2, \ldots
$$

Then there exist $\epsilon>0$ and a neighborhood $U$ of $p$ in $S$ such that the map

$$
f:(-\epsilon, \epsilon) \times U \rightarrow M, \quad f(t, x)=\psi_{t}(x),
$$

is a minimal immersion.

(b) If, in addition to the above assumptions, $M$ is nonpositively curved, globally symmetric and simply connected $[1$ and $S$ is complete, then the map $f$ extends to a complete minimal embedding $\mathbf{R} \times S \rightarrow M$.

Remark 2.2. Let $\mathfrak{p}$ be the set of all infinitesimal transvections at $p$, and let $\mathfrak{k}$ be the Lie algebra of the local isotropy group at $p$. Then $\mathfrak{g}=\mathfrak{k}+\mathfrak{p}$ is a Lie algebra of vector fields on a neighborhood of $p$, and it is the involutive Lie algebra associated to $M$ at $p$ (see Wol74, section 8.1). Recall that every infinitesimal transvection at $p$ extends some vector in $T_{p} M$, and this defines an identification of $\mathfrak{p}$ with $T_{p} M$; in particular, $\mathfrak{p}$ is equipped with an $\operatorname{ad}_{\mathfrak{k}}$-invariant inner product. Let $\mathfrak{s} \subset \mathfrak{g}$ correspond to $T_{p} S \subset T_{p} M$. Then the property of $S$ being totally geodesic in $M$ corresponds to the fact that $\mathfrak{s} \subset \mathfrak{g}$ is a Lie triple system, namely $[[\mathfrak{s}, \mathfrak{s}], \mathfrak{s}] \subset \mathfrak{s}$. Now the assumption in the theorem can be rewritten as

$$
\left[X, \operatorname{ad}_{Y}^{2 n+1} X\right] \in \mathfrak{s} \quad \text { for } Y \in \mathfrak{s} \text { and } n=0,1,2, \ldots
$$

In applications we shall often refer to this equivalent condition in order to check that the hypothesis of the theorem is fulfilled. Note also that condition (2.1) is in general too weak to imply that $f$ is a totally geodesic immersion, for $\mathfrak{s}+\mathbf{R} X$ need not be a Lie triple system of $\mathfrak{g}$.

Proof. (a) Let $V$ be a normal neighborhood of $p$ in $M$ where the Killing field $X$ is defined. We first show that $X$ is normal to $S$ in $U=S \cap V$. In fact, let $q \in U$ be arbitrary and let $c(t), 0 \leq t \leq 1$, be a geodesic segment in $U$ joining $p$ to $q$.

\footnotetext{
${ }^{1}$ Simple connectedness is automatic if $M$ is negatively curved and globally symmetric ([Wol74], Cor. 8.3.13).
} 
Take any parallel vector field $Y(t)$ along $c(t)$ that is everywhere tangent to $S$, and define the function $g(t)=\langle X, Y\rangle_{c(t)}$. We have that $g$ is a real analytic function, because $M$ is an analytic Riemannian manifold (see Proposition 5.5 in Hel78). Since $X$ is orthogonal to $S$ at $p$, we also have that $g(0)=0$. Consider the linear operator $\mathcal{J}: T_{p} M \rightarrow T_{p} M$ defined by $\mathcal{J}(v)=R\left(c^{\prime}(0), v\right) c^{\prime}(0)$. The restriction of $X$ to a vector field along $c, X(t)$, is a Jacobi field; therefore $X^{\prime \prime}(0)=\mathcal{J}(X(0))$. Next we use the facts that $\mathcal{J}$ is a symmetric operator with respect to the inner product in $T_{p} M, T_{p} S$ is a $\mathcal{J}$-invariant subspace (because $S$ is totally geodesic in $M), c^{\prime \prime}=Y^{\prime}=0, \nabla R=0$ (because $M$ is locally symmetric) and $X^{\prime}(0)=0$ (because $X$ is an infinitesimal transvection at $p$ ) to compute that

$$
g^{(2 n)}(0)=\left\langle\mathcal{J}^{n}(Y(0)), X(0)\right\rangle=0
$$

and

$$
g^{(2 n+1)}(0)=\left\langle\mathcal{J}^{n}(Y(0)), X^{\prime}(0)\right\rangle=0 \quad \text { for } n=0,1,2, \ldots
$$

Since the derivatives of all orders of $g$ vanish at 0 , it follows that $g$ identically vanishes. Therefore $\langle X, Y\rangle_{c(1)}=g(1)=0$. Since $Y(1)$ can be any vector in $T_{q} S$, this proves that $X$ is orthogonal to $S$ at $q$.

We have that, $\left\{\psi_{t}\right\}$ being a local one-parameter group of isometries, there exists $\epsilon>0$ such that $f(t, x)$ is well-defined and satisfies $f(t, x)=\left(\psi_{t} \circ f\right)(0, x)$ for $(t, x) \in(-\epsilon, \epsilon) \times U$. Note that, for any $x \in U$, the image of the differential of $f$ at $(0, x)$ equals the subspace $\mathbf{R} X_{x}+T_{x} S$, where $X_{x}$ is normal to $T_{x} S$; so $f$ is an immersion at $(0, x)$ if and only if $X_{x} \neq 0$. It is then clear that we can shrink $U$, if necessary, so that $f$ is an immersion on $(-\epsilon, \epsilon) \times U$. Moreover, in order to see that $f$ is a minimal immersion, it is enough to compute the mean curvature vector of $f$ for $t=0$. Let $q \in U$, and let $n$ be a normal unit vector field along $f$ near $f(0, q)=q$. If $v \in T_{q} S$, then $\left\langle\nabla_{v} n, v\right\rangle_{q}=0$, because $n$ is normal to $S$ and $S$ is totally geodesic. Since $X_{q} \perp T_{q} S$ and $X_{q} \neq 0$, we only need to check that $\left\langle\nabla_{\frac{X}{\|X\|}} n, \frac{X}{\|X\|_{q}}\right\rangle_{q}=-\frac{1}{\|X\|^{2}}\left\langle n, \nabla_{X} X\right\rangle_{q}=0$.

So next we show that $\left(\nabla_{X} X\right)_{q} \in T_{q} S$. Consider the involutive Lie algebra $\mathfrak{g}=\mathfrak{k}+$ $\mathfrak{p}$ associated to $M$ at $p$ as in the remark. There exists $Y \in T_{p} S$ such that $q=\exp _{p} Y$. Let $\varphi$ be the local transvection from $p$ to $q$ along $t \mapsto \exp _{p} t Y$. Then $\varphi(p)=q$; the differential $d \varphi_{p}: T_{p} M \rightarrow T_{q} M$ is parallel transport along $t \mapsto \exp _{p} t Y$ and maps $T_{p} S$ onto $T_{q} S$. Moreover, $\left(\nabla_{X} X\right)_{q}=d \varphi_{p}\left(\nabla_{Z} Z\right)_{p}$, where $Z=\varphi_{*}^{-1} X$. So it is enough to verify that $\left(\nabla_{Z} Z\right)_{p} \in T_{p} S$.

Note that the local flow defined by $Z$ is $\left\{\varphi^{-1} \psi_{t} \varphi\right\}$ and, on the Lie group level, we have that $\varphi^{-1} \psi_{t} \varphi=\exp t \operatorname{Ad}_{\varphi^{-1}} X$. So

$$
Z=\operatorname{Ad}_{\varphi^{-1}} X=e^{-\operatorname{ad}_{Y}} \cdot X=Z^{\mathfrak{k}}+Z^{\mathfrak{p}},
$$

where

$$
Z^{\mathfrak{k}}=\sum_{n=0}^{\infty} \frac{1}{(2 n) !} \operatorname{ad}_{Y}^{2 n} X \quad \text { and } \quad Z^{\mathfrak{p}}=-\sum_{m=0}^{\infty} \frac{1}{(2 m+1) !} \operatorname{ad}_{Y}^{2 m+1} X
$$

are respectively the $\mathfrak{k}$ and $\mathfrak{p}$ components of $Z \in \mathfrak{g}$ with respect to the decomposition $\mathfrak{g}=\mathfrak{k}+\mathfrak{p}$. According to results in ch. X, section 2 and ch. XI, section 3 of [KN69] we have that $\left(\nabla_{Z} Z\right)_{p}=\left[Z^{\mathfrak{k}}, Z^{\mathfrak{p}}\right]$. Therefore,

$$
\left(\nabla_{Z} Z\right)_{p}=-\sum_{n=0}^{\infty} \sum_{m=0}^{\infty} \frac{1}{(2 n !)(2 m+1) !}\left[\operatorname{ad}_{Y}^{2 n} X, \operatorname{ad}_{Y}^{2 m+1} X\right] .
$$


Our claim that $\left(\nabla_{Z} Z\right)_{p} \in T_{p} S$ is now a consequence of condition (2.1) and the lemma below. This completes the proof that $f$ is a minimal immersion.

(b) Since here $M$ is globally symmetric, we have that the Killing field $X$ is globally defined, and $\psi_{t}$ is defined for all $t \in \mathbf{R}$ and it is a global isometry; so $f$ is well-defined on $\mathbf{R} \times S$. We claim that $f$ is an immersion everywhere. It suffices to check this at a point $(0, q) \in \mathbf{R} \times S$. Since the restriction of $X$ to the geodesic connecting $p$ and $q$ is a nontrivial Jacobi field and $M$ is nonpositively curved, we get that $X_{q}$ is not zero. This implies that $f$ is an immersion at $(0, q)$.

We next show that $f$ is injective. Note that this is equivalent to having $\psi_{t}(S) \cap$ $S=\varnothing$ for $t \neq 0$. In the following we prove in fact that $d\left(S, \psi_{t}(S)\right)=|t||| X_{p}||$, where $d$ is the metric distance on $M$. Let $\gamma$ be the geodesic in $M$ through $p$ with initial velocity $X_{p}$. Since $S$ is totally geodesic in $M$ and $M$ is nonpositively curved, the function $\rho: x \mapsto d^{2}(x, S)$ is convex on $M$ (see [BO69]). But $T=\psi_{t}(S)$ is totally geodesic; so the restriction $\left.\rho\right|_{T}$ is also convex. Now $\gamma$ is a common perpendicular from $S$ to $T$. So a first variation argument shows that $\gamma(t) \in T$ is a critical point of $\left.\rho\right|_{T}$. Thus $\gamma(t)$ must be global minimum point of $\rho_{T}$. Clearly $\gamma(t) \notin S$, and there cannot be another perpendicular from $\gamma(t)$ to $S$ besides $\gamma$, for otherwise we would have a geodesic triangle with two right angles in $M$, which is impossible since $M$ is nonpositively curved. Hence $d(S, T)=d(\gamma(0), \gamma(t))=|t|\left\|X_{p}\right\|$.

We finally prove that $f$ is a proper map. Let $\left\{\left(t_{n}, x_{n}\right)\right\}$ be a sequence in $\mathbf{R} \times S$ such that $\left(t_{n}, x_{n}\right) \rightarrow \infty$. (If $\left\{a_{n}\right\}$ is a sequence in a topological space $A$, the notation $a_{n} \rightarrow \infty$ means that the sequence eventually leaves any given compact subset of $A$.) We need to prove that $f\left(t_{n}, x_{n}\right) \rightarrow \infty$. Since $\mathbf{R} \times S$ has the product topology, we have that either $t_{n} \rightarrow \infty$ or $x_{n} \rightarrow \infty$. In the former case, as we have seen above, $d\left(p, \psi_{t}(x)\right) \geq|t||| X_{p}||$ for $x \in S$, and this implies that $f\left(t_{n}, x_{n}\right) \rightarrow \infty$. In the latter case, $f\left(t_{n}, x_{n}\right) \rightarrow \infty$ is an easy consequence of the fact that for every $q \in S$ the map $t \mapsto d\left(p, \psi_{t}(q)\right)$ has a global minimum for $t=0$, which can be seen as follows. Of course we may assume that $q \neq p$. If this map has a minimum for some $t \neq 0$, then the points $p, q$ and $\psi_{t}(q)$ specify a geodesic triangle with two right angles, contradicting the fact that $M$ is nonpositively curved. Therefore the minimum is attained for $t=0$. This completes the proof of the theorem.

Lemma 2.3. Let $Y \in \mathfrak{s}$. If $\left[X, \operatorname{ad}_{Y}^{2 m+1} X\right] \in \mathfrak{s}$ for all $m \geq 0$, then we have that $\left[\operatorname{ad}_{Y}^{2 n} X, \operatorname{ad}_{Y}^{2 m+1} X\right] \in \mathfrak{s}$ for all $n, m \geq 0$.

Proof. We prove by induction on $n+m$ that

$$
\operatorname{ad}_{Y}\left[\operatorname{ad}_{Y}^{2 n} X, \operatorname{ad}_{Y}^{2 m} X\right] \in \mathfrak{s} \text { and }\left[\operatorname{ad}_{Y}^{2 n} X, \operatorname{ad}_{Y}^{2 m+1} X\right] \in \mathfrak{s} .
$$

In the initial case of $n+m=0$ we must have $n=m=0$, and then the assertion is clear.

Let $n, m \geq 0$ be such that $n+m>0$. We first show that $\operatorname{ad}_{Y}\left[\operatorname{ad}_{Y}^{2 n} X, \operatorname{ad}_{Y}^{2 m} X\right] \in$ $\mathfrak{s}$. Without loss of generality, we may assume that $n \geq m$. We proceed by induction on $n-m$. The case $n-m=0$ is trivial. Consider the case $n-m=1$ and write, via the Jacobi identity,

$$
\operatorname{ad}_{Y}\left[\operatorname{ad}_{Y}^{2 n} X, \operatorname{ad}_{Y}^{2 m} X\right]=\operatorname{ad}_{Y}^{2}\left[\operatorname{ad}_{Y}^{2 n-1} X, \operatorname{ad}_{Y}^{2 m} X\right]-\operatorname{ad}_{Y}\left[\operatorname{ad}_{Y}^{2 n-1} X, \operatorname{ad}_{Y}^{2 m+1} X\right] .
$$

The first summand of the right-hand side belongs to $\mathfrak{s}$ by the induction hypothesis on $n+m$ (and the fact that $\operatorname{ad}_{Y}^{2} \mathfrak{s} \subset \mathfrak{s}$, since $\mathfrak{s}$ is a Lie triple system), and the second summand is zero because $n-m=1$. Therefore $\operatorname{ad}_{Y}\left[\operatorname{ad}_{Y}^{2 n} X, \operatorname{ad}_{Y}^{2 m} X\right] \in \mathfrak{s}$. 
Now assume $n-m>1$ and write

$$
\begin{aligned}
\operatorname{ad}_{Y}\left[\operatorname{ad}_{Y}^{2 n} X, \operatorname{ad}_{Y}^{2 m} X\right] & =\operatorname{ad}_{Y}^{2}\left[\operatorname{ad}_{Y}^{2 n-1} X, \operatorname{ad}_{Y}^{2 m} X\right] \\
& \quad-\operatorname{ad}_{Y}^{2}\left[\operatorname{ad}_{Y}^{2 n-2} X, \operatorname{ad}_{Y}^{2 m+1} X\right]+\operatorname{ad}_{Y}\left[\operatorname{ad}_{Y}^{2 n-2} X, \operatorname{ad}_{Y}^{2 m+2} X\right] .
\end{aligned}
$$

The first two summands of the right-hand side belong to $\mathfrak{s}$ by the induction hypothesis on $n+m$, and the last summand belongs to $\mathfrak{s}$ by the induction hypothesis on $n-m$. Therefore $\operatorname{ad}_{Y}\left[\operatorname{ad}_{Y}^{2 n} X, \operatorname{ad}_{Y}^{2 m} X\right] \in \mathfrak{s}$.

Next we show that $\left[\operatorname{ad}_{Y}^{2 n} X, \operatorname{ad}_{Y}^{2 m+1} X\right] \in \mathfrak{s}$. For this purpose we proceed by induction on $n$. The case $n=0$ is precisely the assumption of the lemma. Now assume $n>0$ and write

$$
\begin{aligned}
{\left[\operatorname{ad}_{Y}^{2 n} X, \operatorname{ad}_{Y}^{2 m+1} X\right] } & =\operatorname{ad}_{Y}^{2}\left[\operatorname{ad}_{Y}^{2 n-2} X, \operatorname{ad}_{Y}^{2 m+1} X\right] \\
-2 \operatorname{ad}_{Y} & {\left[\operatorname{ad}_{Y}^{2 n-2} X, \operatorname{ad}_{Y}^{2 m+2} X\right]+\left[\operatorname{ad}_{Y}^{2 n-2} X, \operatorname{ad}_{Y}^{2 m+3} X\right] . }
\end{aligned}
$$

The first summand of the right-hand side belongs to $\mathfrak{s}$ by the induction hypothesis on $n+m$, the second summand belongs to $\mathfrak{s}$ by the arguments in the previous paragraph, and the last summand belongs to $\mathfrak{s}$ by the induction hypothesis on $n$. Therefore $\left[\operatorname{ad}_{Y}^{2 n} X, \operatorname{ad}_{Y}^{2 m+1} X\right] \in \mathfrak{s}$, and this completes the induction step on $n+$ $m$.

\section{Applications}

In this section we describe some instances where the theorem can be applied. The most interesting case is probably that of the complex hyperbolic plane $\mathbf{H}^{2}(\mathbf{C})$, which accomodates two classes of minimal hypersurfaces that are constructed by making use of the theorem.

3.1. Reflective submanifolds. An embedded submanifold $B$ of a complete Riemannian manifold $M$ is called reflective if $B$ is complete with respect to the induced metric and it is a connected component of the fixed point set of an involutive isometry of $M$ (see [Leu73]). By a well-known result about fixed points of isometries, every reflective submanifold is automatically totally geodesic. In case $M$ is a simply connected globally symmetric space and $\mathfrak{g}=\mathfrak{k}+\mathfrak{p}$ is the associated involutive Lie algebra with respect to some fixed base point, the reflective submanifolds $B$ through the base point are in bijective correspondence with the subspaces $\mathfrak{b}$ of $\mathfrak{p}$ such that $\mathfrak{b}$ and its orthogonal complement $\mathfrak{b}^{\perp}$ are Lie triple systems and

$$
\left[\left[\mathfrak{b}, \mathfrak{b}^{\perp}\right], \mathfrak{b}\right] \subset \mathfrak{b}^{\perp}, \quad\left[\left[\mathfrak{b}, \mathfrak{b}^{\perp}\right], \mathfrak{b}^{\perp}\right] \subset \mathfrak{b} .
$$

Such a $\mathfrak{b}$ is called a reflective subspace of $\mathfrak{p}$.

If $B$ is a reflective submanifold of a nonpositively curved simply connected globally symmetric space $M$ and $v$ is any nonzero normal vector to $B$, then it is readily seen that the pair $(B, v)$ satisfies condition (2.1). This shows that reflective submanifolds in nonpositively curved simply connected globally symmetric spaces may be translated along any normal geodesic to produce a minimal submanifold, thereby providing an extensive number of situations to which our theorem can be applied. See [Leu74, Leu79a] for tables with the classification of reflective submanifolds in symmetric spaces. In the following we will comment on two interesting particular cases. 
3.1.1. Rank one symmetric spaces. Every totally geodesic submanifold of a rank one globally symmetric space $M$ is reflective (Leu74, Leu79a]). We have that the maximal totally geodesic submanifolds of $\mathbf{H}^{n}(\mathbf{C})$ are $\mathbf{H}^{n-1}(\mathbf{C})$ and $\mathbf{H}^{n}(\mathbf{R})$; those of $\mathbf{H}^{n}(\mathbf{H})$ (quaternionic hyperbolic space) are $\mathbf{H}^{n-1}(\mathbf{H})$ and $\mathbf{H}^{n}(\mathbf{C})$; and those of $\mathbf{H}^{2}$ (Cay) (Cayley hyperbolic plane) are $\mathbf{H}^{2}(\mathbf{H})$ and $\mathbf{H}^{8}(\mathbf{R})$ (Wol63]). In each case, the isotropy subgroup of the totally geodesic submanifold at the base point acts transitively on the unit sphere of the normal space at that point. Corresponding to each above-listed type of totally geodesic submanifold in $M$, the construction thus provides precisely one congruence class of minimal embeddings $\mathbf{R}^{N} \rightarrow M$, irrespective of the choice of normal vector.

In particular, if $M=\mathbf{H}^{n}(\mathbf{C})$, then the construction provides minimal embeddings $\mathbf{R}^{2 n-1} \rightarrow \mathbf{H}^{n}(\mathbf{C})$ and $\mathbf{R}^{n+1} \rightarrow \mathbf{H}^{n}(\mathbf{C})$. The former submanifold is easily seen to be a bisector (see Gol99]), whereas the latter one is a new example of a minimal submanifold in $\mathbf{H}^{n}(\mathbf{C})$, and it is a hypersurface if $n=2$.

3.1.2. Real forms of Hermitian symmetric spaces. A particularly interesting class of reflective submanifolds is composed of the fixed point sets of involutive antiholomorphic isometries of Hermitian symmetric spaces (which are automatically connected, see [Leu79b]). Such reflective submanifolds are called real forms of the underlying Hermitian symmetric spaces, and correspond precisely to the reflective subspaces $\mathfrak{b}$ (cf. supra) which are totally real subspaces of $\mathfrak{p}$. The classification of real forms of Hermitian symmetric spaces is given in Leu79b. It is interesting to note that we recover $\mathbf{H}^{n}(\mathbf{R})$ as a real form of $\mathbf{H}^{n}(\mathbf{C})$.

3.2. Other examples. We now use the restricted root decomposition of a real semisimple Lie algebra (see [Hel78], $\S 3$, ch. VI) in order to show that there are examples of totally geodesic submanifolds in symmetric spaces that are not necessarily reflective but nevertheless can be translated along some normal geodesic to produce a minimal submanifold. Let $\mathfrak{g}=\mathfrak{k}+\mathfrak{p}$ be the involutive Lie algebra associated to a symmetric space, and denote the involution by $\theta$. Choose a maximal Abelian subspace $\mathfrak{a} \subset \mathfrak{p}$. Then there is an $\operatorname{ad}_{\mathfrak{a}}$-invariant decomposition $\mathfrak{g}=\mathfrak{m}+\mathfrak{a}+\sum_{\lambda \in \Sigma} \mathfrak{g}_{\lambda}$, where $\mathfrak{m}$ is the centralizer of $\mathfrak{a}$ in $\mathfrak{k}, \Sigma$ is the restricted root system and, for each $\lambda \in \Sigma, \mathfrak{g}_{\lambda}$ is the corresponding restricted root space. Since $\theta\left[\mathfrak{g}_{\lambda}\right]=\mathfrak{g}_{-\lambda}$, we can write

$$
\mathfrak{k}=\mathfrak{m}+\sum_{\lambda \in \Sigma^{+}} \mathfrak{k}_{\lambda} \quad \text { and } \quad \mathfrak{p}=\mathfrak{a}+\sum_{\lambda \in \Sigma^{+}} \mathfrak{p}_{\lambda}
$$

where $\mathfrak{k}_{\lambda}=\mathfrak{k} \cap\left(\mathfrak{g}_{\lambda}+\mathfrak{g}_{-\lambda}\right), \mathfrak{p}_{\lambda}=\mathfrak{p} \cap\left(\mathfrak{g}_{\lambda}+\mathfrak{g}_{-\lambda}\right)$ and $\Sigma^{+}$is the positive restricted root system. Now fix any $\lambda \in \Sigma^{+}$, define $\mathfrak{s}=\mathfrak{p}_{\lambda}$ and choose $X \in \mathfrak{a}$. We claim that $\mathfrak{s}$ is a Lie triple system and the pair $(\mathfrak{s}, X)$ satisfies condition (2.1). This is to be a consequence of the following commutation rules (see [Hel78], Lemma 11.4, ch. VII):

$$
\left[\mathfrak{k}_{\lambda}, \mathfrak{p}_{\mu}\right] \subset \mathfrak{p}_{\lambda+\mu}+\mathfrak{p}_{\lambda-\mu}, \quad\left[\mathfrak{k}_{\lambda}, \mathfrak{k}_{\mu}\right] \subset \mathfrak{k}_{\lambda+\mu}+\mathfrak{k}_{\lambda-\mu}, \quad\left[\mathfrak{p}_{\lambda}, \mathfrak{p}_{\mu}\right] \subset \mathfrak{k}_{\lambda+\mu}+\mathfrak{k}_{\lambda-\mu} .
$$

In fact, $\left[\mathfrak{p}_{\lambda},\left[\mathfrak{p}_{\lambda}, \mathfrak{p}_{\lambda}\right]\right] \subset\left[\mathfrak{p}_{\lambda}, \mathfrak{k}_{2 \lambda}+\mathfrak{m}\right] \subset \mathfrak{p}_{\lambda} ;$ so $\mathfrak{p}_{\lambda}$ is a Lie triple system. Moreover, for any $Y \in \mathfrak{p}_{\lambda}$ we have that $\operatorname{ad}_{Y}^{2 n+1} X \in \mathfrak{k}_{\lambda}$ and $\operatorname{ad}_{Y}^{2 n} X \in \mathfrak{a}+\mathfrak{p}_{2 \lambda}$ for $n=0,1,2, \ldots$. Therefore $\left[X, \operatorname{ad}_{Y}^{2 n} X\right] \in \mathfrak{p}_{\lambda}$ for $n=0,1,2, \ldots$. 


\section{REFERENCES}

[BO69] R. L. Bishop and B. O'Neill, Manifolds of negative curvature, Trans. Amer. Math. Soc. 145 (1969), 1-49. MR 40:4891

[Gol99] W. M. Goldman, Complex hyperbolic geometry, Oxford Mathematical Monographs, The Clarendon Press, New York, 1999. MR 2000g:32029

[Hel78] S. Helgason, Differential geometry, Lie groups, and symmetric spaces, Pure and Applied Mathematics, vol. 80, Academic Press, New York - London, 1978. MR 80k:53081

[KN69] S. Kobayashi and K. Nomizu, Foundations of differential geometry, Wiley Interscience Publishers, vol. 1 (1963) and vol. 2 (1969). MR 27:2945 MR 38:6501

[Leu73] D. S. P. Leung, The reflection principle for minimal submanifolds of Riemannian symmetric spaces, J. Differential Geom. 8 (1973), 153-160. MR 51:4114

[Leu74] D. S. P. Leung, On the classification of reflective submanifolds of Riemannian symmetric spaces, Indiana Univ. Math. J. 24 (1974), 327-339, Errata in same volume, p. 1199. MR 51:4115 MR 51:13935

[Leu79a] D. S. P. Leung, Reflective submanifolds III: congruence of isometric reflective submanifolds and corrigenda to the classification of reflective submanifolds, J. Differential Geom. 14 (1979), 167-177. MR 83e:53052

[Leu79b] D. S. P. Leung, Reflective submanifolds IV: classification of real forms of Hermitian symmetric spaces, J. Differential Geom. 14 (1979), 179-185. MR 83e:53053

[Wol63] J. A. Wolf, Elliptic spaces in Grassmann manifolds, Illinois J. Math. 7 (1963), 447-462. MR 27:6221

[Wol74] J. A. Wolf, Spaces of constant curvature, Publish or Perish, Boston, 1974. MR 49:7958

Instituto de Matemática e Estatística, Universidade de São Paulo, Rua do Matão, 1010, SÃo Paulo, SP 05508-090, BRaZIL

E-mail address: gorodski@ime.usp.br 